\title{
Intraoperative Life Threatening Acute Lung Injury Due to Multiple Septic Pulmonary Emboli during Transpedicular Biopsy and Kyphoplasty
}

\author{
Karuppaiah Karthik ${ }^{1}$, Ajoy Prasad Shetty ${ }^{2}$, Shanmuganathan Rajasekaran ${ }^{2}$ \\ ${ }^{1}$ Department of Orthopaedic Surgery, King's College Hospital, Denmark Hill, London, UK \\ ${ }^{2}$ Department of Orthopaedics, Traumatology and Spine Surgery, Ganga Hospital, Coimbatore, Tamilnadu, India
}

\begin{abstract}
A 55-year-old gentleman was presented to our clinic two months after a trivial fall with persistent pain, gibbus at the thoraco-lumbar junction and intact neurology. Radiological and laboratory investigations suggested osteoporotic fracture or metastasis. Due to unremitting pain, a plan was made to do transpedicular biopsy and kyphoplasty. Biopsy needles were inserted into both pedicles and an attempt at aspiration was made. Since the aspirate was dry, 5 to $6 \mathrm{~mL}$ of saline was injected through one needle and an attempt at aspiration was made through the other. Three-millilitres of sero-sanguineous material mixed with pus came out and kyphoplasty was deferred. After extubation, the patient developed severe bronchospasm and was transferred to the intensive care unit. Investigations confirmed multiple septic pulmonary emboli and the patient recovered completely after treatment. This report highlights that confirmation of the diagnosis is essential before performing any procedure that increases the intravertebral pressure and the place should have appropriate facilities to manage complications.
\end{abstract}

Keywords: Transpedicular biopsy; Kyphoplasty; Septic pulmonary emboli; Acute lung injury; Infective spondylitis

\section{Introduction}

A transpedicular biopsy is an important tool in the evaluation of vertebral body lesions [1,2]. It can be performed with minimal morbidity and high diagnostic yield as an outpatient procedure $[1,2]$. Though minor complications have been reported, major complications related to perivertebal venous leaks have not been reported in the literature. Increased intra vertebral pressure can cause embolism from the vertebral venous sinuses in the pulmonary and systemic circulation [3]. We report a case of vertebral osteomyelitis causing intraoperative multiple septic pulmonary emboli leading to acute lung injury during transpedicular biopsy and kyphoplasty.

\section{Case Report}

This report highlights this rare life threatening intraoperative complication, following transpedicular biopsy and kyphoplasty. A 55-year-old gentleman experienced a trivial fall and developed severe back pain. He had some analgesics and bed rest for a few days, which relieved the pain. He then started performing his routine activities. The back pain gradually increased and incapacitated him from doing his routine day-to-day activities. The patient came to our clinic two months after the fall with com-

Received Oct 8, 2012; Revised Apr 23, 2013; Accepted May 1, 2013

Corresponding author: Karuppaiah Karthik

Department of Orthopaedic Surgery, King's College Hospital,

Denmark Hill, London SE5 9RS, UK

Tel: +44-7542011723, Fax: +44-1642282784, E-mail: karthi97@gmail.com 
plaints of persistent back pain and restriction of movements.

On examination, the patient had gibbus at the thoracolumbar junction with spasm of the para-spinal muscles. The movements of the spine were restricted and painful. The motor power and sensation distally was normal with intact reflexes. The patient also had normal bladder and bowel function. The other positive findings were anorexia and loss of weight in the preceding two months. $\mathrm{He}$ is also a well-controlled type-2 diabetic on oral medications for the past four years. He is not on any other medication and is not allergic to anything that he knows of. The

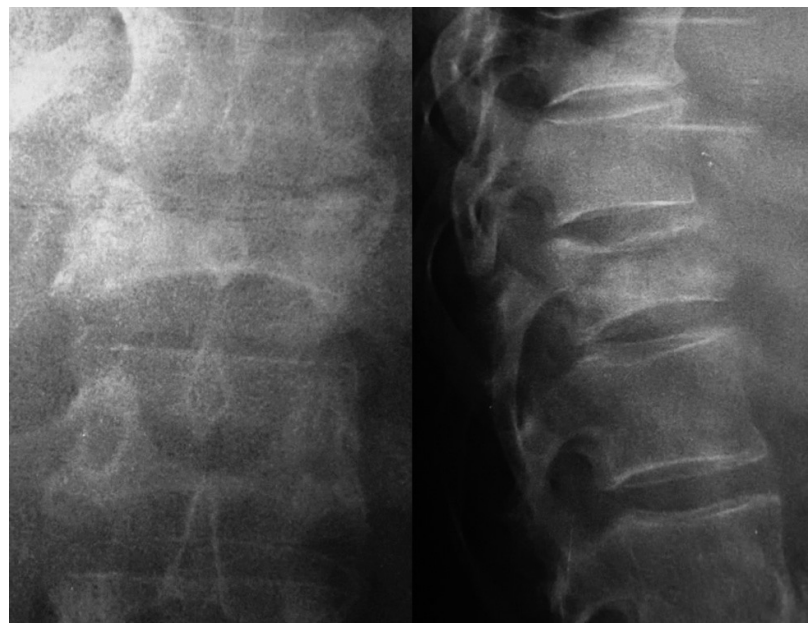

Fig. 1. The antero-posterior and lateral view of X-rays showed compression fracture of $\mathrm{T} 12$ vertebra with normal disc space. patient's cardiac and pulmonary status is good with no other constitutional symptoms. The patient had a surgical fixation of the fracture of his humerus 10 years back under general anaesthesia with uneventful postoperative recovery. He is not a smoker or an alcoholic. The patient also had no history of any childhood illnesses.

Blood investigations showed slightly raised C-reactive protein (CRP) $11 \mathrm{mg} / \mathrm{L}$ (normal, $<5 \mathrm{mg} / \mathrm{L}$ ), erythrocyte sedimentation rate (ESR) $27 \mathrm{~mm} / \mathrm{hr}$ (normal, $0-25 \mathrm{~mm} /$ $\mathrm{hr}$ ), and high normal white cell count 10,000 cells $/ \mathrm{mm}^{3}$ (normal, 4,000-11,000 cells $/ \mathrm{mm}^{3}$ ). Other investigations were normal, including the bone profile, renal and liver function test. The X-ray showed compression fracture of the T12 vertebra with normal disc space (Fig. 1). Magnetic resonance imaging (MRI) showed marrow edema with evidence suggestive of fluid in the fracture site (Fig. 2). The cord was normal with normal signal intensity from the intervertebral discs. MRI also showed prevertebral soft-tissue swelling and a subtle amount of the epidural soft-tissue component indenting the anterior dural sac. These findings were discussed in a multi-disciplinary meeting and a differential diagnosis of tumour that may be primary or secondary, compression fracture with pseudoarthrosis and infection possibly body type tuberculosis as there is still an endemic for tuberculosis in India. The patient was then investigated for the possibility of tumour elsewhere. Both the hematological and radiological investigations, including the bone scan was not

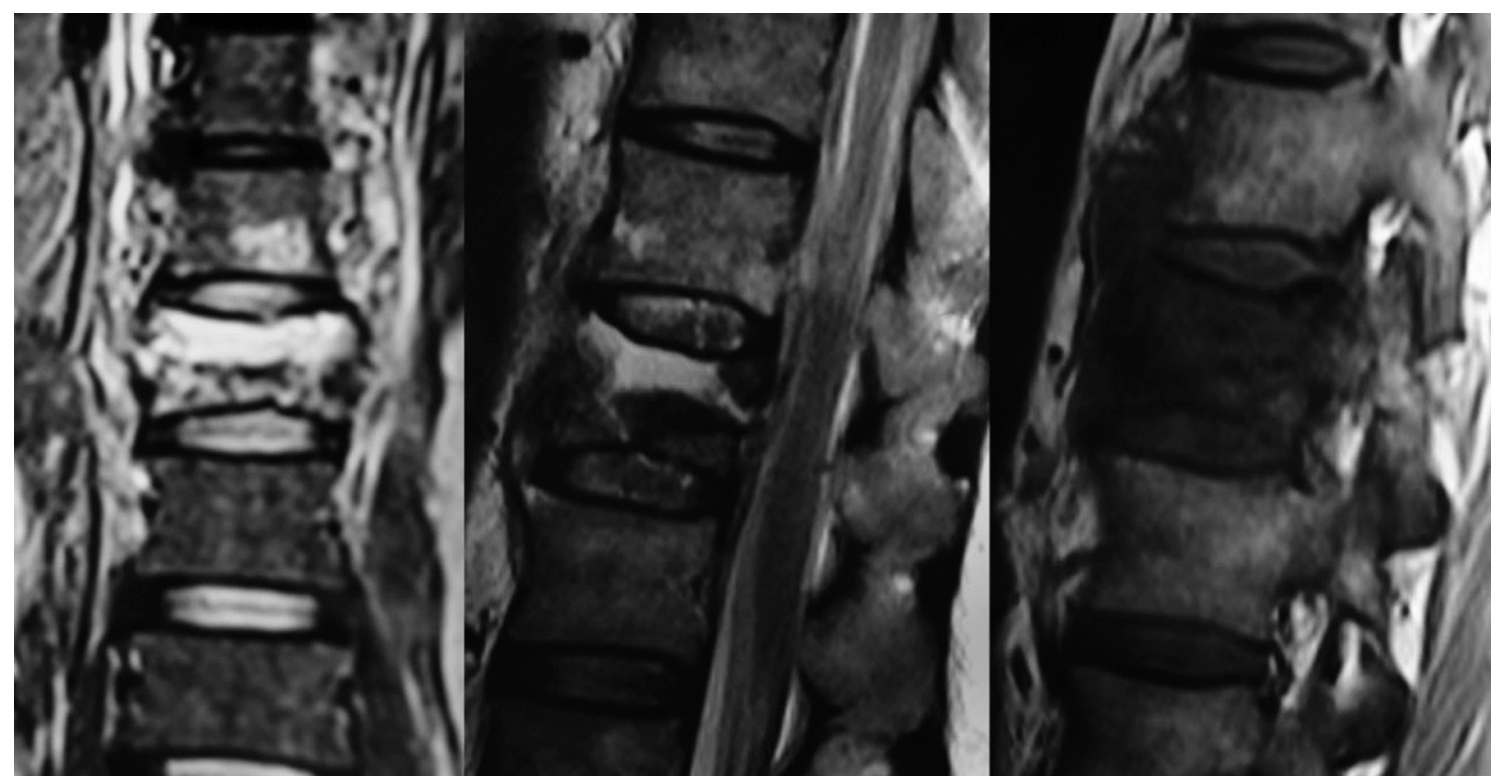

Fig. 2. Magnetic resonance imaging (MRI) showed marrow edema with evidence suggestive of fluid in the fracture site (pseudoarthrosis). The cord was normal with normal signal intensity from the intervertebral discs. MRI also showed prevertebral soft-tissue swelling and a subtle amount of epidural soft-tissue component indenting the anterior dural sac. 
suggestive of any abnormality elsewhere in the body. The dual-energy X-ray absorptiometry scan showed that the patient is osteopenic with a T-score of -2 . The computed tomography (CT) scan of the T12 vertebra showed intact posterior cortex (Fig. 3).

Since the patient had severe unremitting pain with diagnosis more towards osteoporotic fracture or metastasis, a decision was made to perform transpedicular biopsy and kyphoplasty. The surgery was performed under general anaesthesia with the patient in prone position on Relton Hall frame. Transpedicular biopsy needles were inserted into the pedicles of D12 vertebral body under image control. There was minimal resistance for passage of the needle once it entered the vertebral body and no material came initially on aspiration of both needles on either pedicle. Five to six millilitres of saline was injected through one needle with some pressure and an attempt at aspiration was made through the needle in the other pedicle. Only three millilitres of sero-sanguineous material mixed with pus came out through the second needle. The aspirate was sent for microbiology, cytology and fluid

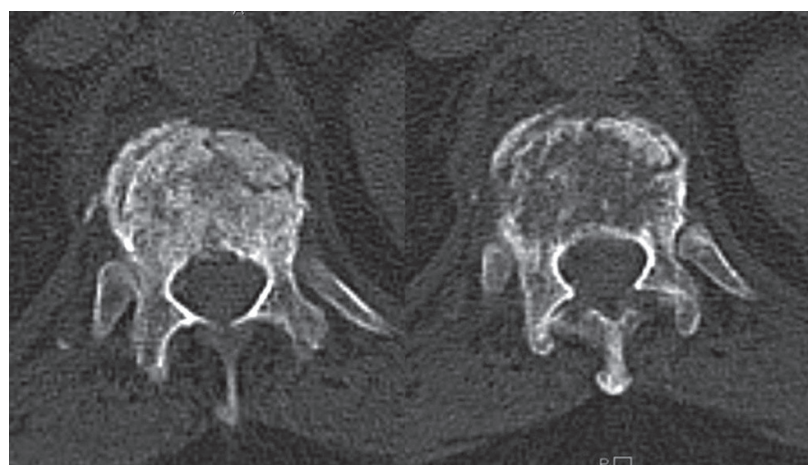

Fig. 3. The computed tomography scan of the T12 vertebra showed intact posterior cortex. analysis. Since the aspirate suggested the possibility of infection, kyphoplasty was deferred.

The patient was turned in supine position and was extubated. Immediately after extubation, the patient went in for severe bronchospasm with decrease in saturation. The patient was unable to maintain his tidal volume and was again intubated. The arterial blood gas analysis showed severe respiratory acidosis with $\mathrm{pH} 7.16, \mathrm{PaCO}_{2} 72 \mathrm{~mm}$ $\mathrm{Hg}$, and $\mathrm{PaO}_{2} 82 \mathrm{~mm} \mathrm{Hg}$. The chest X-ray taken immediately showed bilateral multiple lung infiltrates more on the lower zones and the left side Fig. 4A. The patient was transferred to the intensive care unit (ICU) and continued on mechanical ventilation. The $\mathrm{PaO}_{2} / \mathrm{FiO}_{2}$ ratio was $205 \mathrm{~mm} \mathrm{Hg}$. The X-ray was repeated in the ICU showed a progression of pulmonary edema around the infiltrates (Fig. 4B). The spiral CT scan showed bilateral multifocal infiltrate more in the peripheries with fluid in both pleural cavities (Fig. 5). Meanwhile, the patient was started on intravenous vancomycin. After 24 hours, the patient was gradually improved and weaned off from the ventilator. The endo-tracheal tube was detached 24 hours later

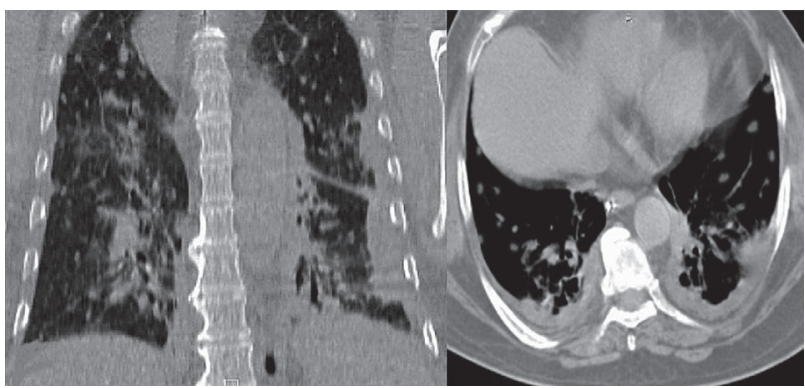

Fig. 5. The spiral computed tomography scan done showed bilateral multifocal infiltrate more in the peripheries with fluid in both pleural cavities.
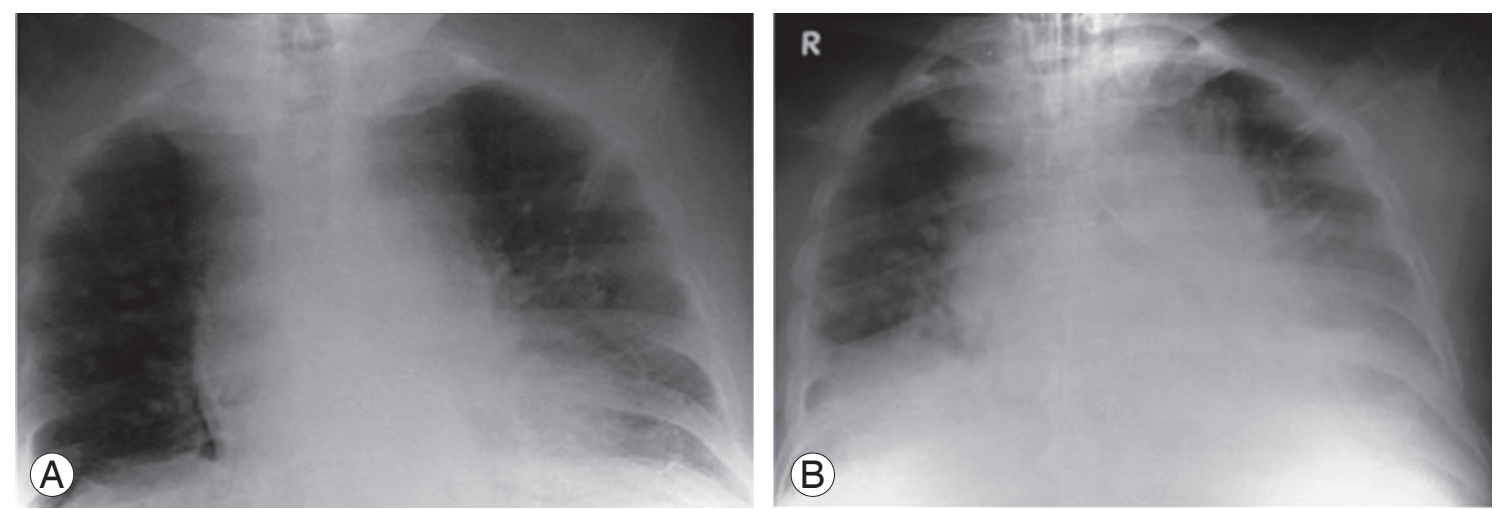

Fig. 4. (A) The chest X-ray taken immediately showed bilateral multiple lung infiltrates more on the lower zones and the left side. (B) The $\mathrm{X}$-ray repeated in intensive care unit showed progression of pulmonary edema around the infiltrates. 
and the patient was kept in a high dependency unit. In 72 hours, the patient's chest was clear and X-ray showed complete resolution of the infiltrates (Fig. 6).

The culture report of the material from D12 vertebra came as Staphylococcus aureus sensitive to imipenum and linezolid. A similar report was obtained from the bronchial lavage aspirate, pleural fluid aspirate and endotracheal tube tip culture. The blood culture was negative. The clinical presentation of the patient followed the case definition of septic pulmonary emboli and included the following: 1) focal or multifocal lung infiltrates compatible with septic embolism to the lung, 2) presence of active extra pulmonary infection as potential embolic source, 3) exclusion of other potential explanation for lung infiltrates, and 4) resolution of lung infiltrates with appropriate antimicrobial therapy.

The patient was then started on intravenous linezolid for two weeks, followed by oral administration for another 6 weeks. The spine was protected with thoracolumbar orthosis for four weeks and was gradually weaned off. The patient recovered uneventfully and the CRP and ESR returned to normal. The patient remained symptom free in his last follow-up at six months.

\section{Discussion}

Sudden intraoperative or perioperative cardio-pulmonary dysfunction due to emboli is a well-known complication

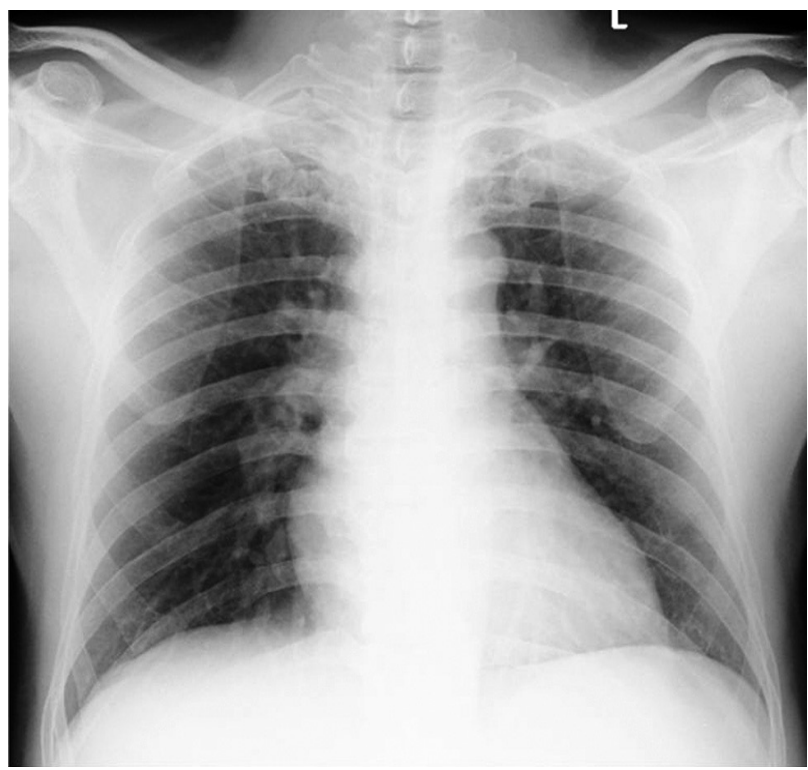

Fig. 6. After administration of antibiotics, the patient's chest was clear and $\mathrm{X}$-ray showed complete resolution of the infiltrates. in bone and joint surgery [4]. There is a sharp rise in the embolic complications in spine surgery after the advent of minimally invasive spine procedures, like vertebroplasty and kyphoplasty; however, these complications after a biopsy were not reported. The difference in the anatomy and (patho)physiology of the vertebral venous system predisposes these procedures to these embolic complications. This system on contrary to the other venous systems is valveless, and is affected by hydrostatic factors, like the change in posture and gravitation forces [3]. Like the azygos veins, this system unites with the superior and inferior vena cava and is protected well within the rigid spinal canal [3]. The blood can flow in either direction depending on the changes of intrathoracic and intraabdominal pressure, and any increase in the intra vertebral pressure can cause embolism directly into the systemic circulation from the vertebral body.

Vertebral osteomyelitis was known to cause infective endocarditis and septic pulmonary emboli, but does it in a chronic fashion leading to long-term disability $[5,6]$. However, in our case, due to the sudden increase in intravertebral pressure, the contents of the vertebra were forced into the systemic circulation. The infected material entered into the pulmonary circulation and caused acute inflammatory reaction in the lung leading to bronchospasm and multiple pulmonary infiltrates. The multiple septic emboli that were lodged in the lung lead to acute lung injury.

Septic pulmonary embolus (SPE) is an uncommon but serious disorder that is difficult to identify [7]. Clinical and radiologic features of SPE at presentation are usually variable and nonspecific, and the diagnosis of this disorder is frequently delayed [7]. The chest X-ray usually shows patchy infiltrates involving peripheral zones of the lower lung lobes $[8,9]$. CT scan demonstrates bilateral multifocal infiltrates, commonly involving the peripheral lung zones, often associated with cavitations $[8,9]$. Prompt recognition of this condition and treatment with antibiotics reduces the complications in these patients.

In our patient, this complication could have been avoided if excess saline was not injected under pressure. However, at some times, where it is necessary to get an aspirate, it is suggested to apply suction through one pedicle, followed by saline injection through the other pedicle. Care should be taken so that the pressure should not be exerted much during injection and there should not be disparity of more than one milliliter between the 
amount injected and aspirated.

A transpedicular biopsy is an image guided minimally invasive procedure that can be performed at low risk if the procedure is performed in a hospital based setting, where qualified surgeons, physicians and ICU facilities are available. A thorough understanding of the anatomy and patho-physiology of the vertebral venous system are essential for the spine surgeons, anesthetists and ICU physicians. Embolic complications can occur even before balloon inflation/cement injection if the intravertebral pressure is increased. The clinical picture will be confusing as various causes are present for this clinical spectrum and radiological appearance of the lung. Early diagnosis of the complications and appropriate management with antibiotics reduces the morbidity and mortality in these patients.

\section{Conflict of Interest}

No potential conflict of interest relevant to this article was reported.

\section{References}

1. Dave BR, Nanda A, Anandjiwala JV. Transpedicular percutaneous biopsy of vertebral body lesions: a series of 71 cases. Spinal Cord 2009;47:384-9.

2. Tehranzadeh J, Tao C, Browning CA. Percutaneous needle biopsy of the spine. Acta Radiol 2007;48:860-8.

3. Groen RJ, du Toit DF, Phillips FM, et al. Anatomical and pathological considerations in percutaneous vertebroplasty and kyphoplasty: a reappraisal of the vertebral venous system. Spine (Phila Pa 1976) 2004;29:1465-71.

4. Takahashi S, Kitagawa H, Ishii T. Intraoperative pulmonary embolism during spinal instrumentation surgery. A prospective study using transoesophageal echocardiography. J Bone Joint Surg Br 2003;85:90-4.

5. Kelly J, Thorning G, Ozzard A, Kelleher K. Back pain and dyspnoea in a middle aged diabetic male. Postgrad Med J 2000;76:239-40,45-8.

6. Lin MY, Rezai K, Schwartz DN. Septic pulmonary emboli and bacteremia associated with deep tissue infections caused by community-acquired methicillin-resistant Staphylococcus aureus. J Clin Microbiol 2008;46:1553-5.

7. Cook RJ, Ashton RW, Aughenbaugh GL, Ryu JH. Septic pulmonary embolism: presenting features and clinical course of 14 patients. Chest 2005;128:162-6.

8. Wong KS, Lin TY, Huang YC, Hsia SH, Yang PH, Chu SM. Clinical and radiographic spectrum of septic pulmonary embolism. Arch Dis Child 2002;87:312-5.

9. Iwasaki Y, Nagata K, Nakanishi M, et al. Spiral CT findings in septic pulmonary emboli. Eur J Radiol 2001;37:190-4. 